\title{
Transmission of Wireless and Wired Services Employing a Simple System Architecture
}

\author{
Wen-Jr Jiang, Chun-Ting Lin, Po-Tsung Shih, Yu-Hung Chen, Jason (Jyehong) Chen, and Sien Chi
}

\begin{abstract}
This letter presents a novel modulation approach for simultaneous generation and transmission of optical radio-frequency (RF) wireless vector signal and baseband (BB) wireline signal using a single-electrode Mach-Zehnder modulator. Based on a double sideband with carrier suppression scheme and properly predistorted driving vector signal, a $2.5-\mathrm{Gb} / \mathrm{s}$ quadrature phase-shift keying (QPSK) signal at $60 \mathrm{GHz}$ is generated with a 1.25-Gb/s BB ON-OFF-keying (OOK) signal. The proposed system can carry higher spectral efficiency vector signals and needs no optical filter to separate the BB and RF signals at remote nodes. Moreover, optical RF signal generation with frequency doubling is also achieved to reduce the bandwidth requirement of the transmitter. After transmission over $25-\mathrm{km}$ single-mode fiber, the power penalties of the generated QPSK and OOK signals are about 4 and $0.1 \mathrm{~dB}$, respectively.
\end{abstract}

Index Terms-Mach-Zehnder modulator (MZM), optical frequency multiplication, optical millimeter-wave, radio-over-fiber (RoF), wavelength-division multiplexer (WDM).

\section{INTRODUCTION}

$\mathbf{T}$ O support the rapidly increasing capacity requirement in broadband access networks, high-frequency wireless system combined with high spectral efficiency modulation formats are widely investigated [1]-[6]. Thanks to the recently released $7-\mathrm{GHz}$ licensed-free band, the $60-\mathrm{GHz}$ system provides a promising solution for the coming high throughput wireless services. However, the transmission distance of the $60-\mathrm{GHz}$ wireless signal is dramatically restricted in a small range due to the high path losses. To extend the coverage of the $60-\mathrm{GHz}$ wireless signals, a $60-\mathrm{GHz}$ radio-over-fiber (RoF) system based on optical fiber networks with unlimited bandwidth and low loss become the key to the next-generation high data rate services [5], [6]. Nevertheless, restricted by the bandwidth of the commercially available $\mathrm{LiNbO}_{3}$ modulators, optical millimeter-wave signal generations with frequency beyond $40 \mathrm{GHz}$ still remain great challenges. On the other hand, high spectral efficiency modulation formats are also required to

Manuscript received August 24, 2009; revised January 07, 2010; accepted January 08, 2010. First published February 02, 2010; current version published March 17, 2010. This work was supported by the National Science Council of the Republic of China, Taiwan, under Contract NSC 98-2218-E-009-021-, Contract NSC 98-2221-E-155-004-MY3, and Contract NSC 97-2221-E-009-105MY3.

W. J. Jiang, P.-T. Shih, Y.-H. Chen, J. Chen are with the Department of Photonics, National Chiao Tung University, Hsinchu 300, Taiwan (e-mail: jiang. eo97g@nctu.edu.tw; jchen@mail.nctu.edu.tw).

C. T. Lin is with the Institute of Photonic System, National Chiao Tung University, Tainan 711, Taiwan (e-mail: jinting@mail.nctu.edu.tw).

S. Chi is with the Department of Photonics Engineering, Yuan Ze University, Chung Li 320, Taiwan.

Color versions of one or more of the figures in this letter are available online at http://ieeexplore.ieee.org.

Digital Object Identifier 10.1109/LPT.2010.2041221

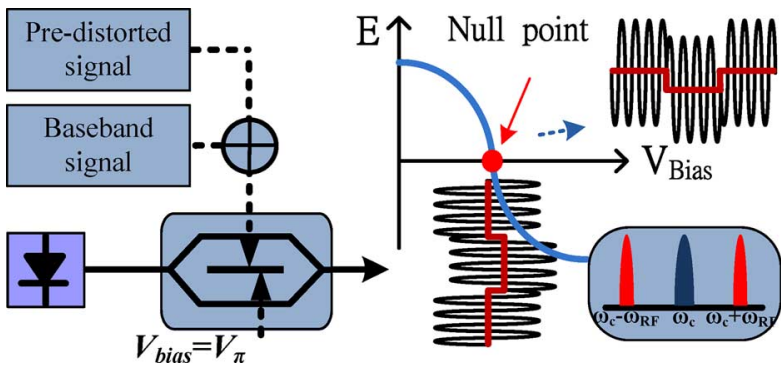

Fig. 1. Concept of proposed system.

transmit high throughput signal within the limited licensed-free band.

In this letter, a $60-\mathrm{GHz}$ multiservices system employing a single electrode Mach-Zehnder modulator (MZM) is experimentally demonstrated. Based on the double sideband with carrier suppression (DSB-CS) scheme, frequency doubling is achieved, and the bandwidth requirement at the transmitter can be reduced. Multilevel modulation format can be generated using a novel signal predistortion method. In addition, a baseband (BB) ON-OFF-keying (OOK) modulation is simultaneously generated and transmitted for wireline applications. No narrowband optical filter which will hinder the implementation in wavelength-division-multiplexing (WDM) system is required at remote node to separate the radio-frequency (RF) and BB signal for different application. After transmission of 25-km standard single-mode fiber (SSMF), the power penalties of $60-\mathrm{GHz}$ quadrature phase-shift keying (QPSK) and OOK signals are less than $4 \mathrm{~dB}$.

\section{CONCEPT AND THEORETICAL ANALYSIS}

Fig. 1 shows the concept of the proposed optical vector signal generation system based on a DSB-CS scheme. The optical field at the input of the single electrode MZM is given by $E_{\text {in }}(t)=E_{o} \cos \left(\omega_{o} t\right)$, where $E_{o}$ and $\omega_{o}$ are the amplitude and angular frequency of the optical field, respectively. The electrical driving signal sent into the MZM is $V(t)=V_{\mathrm{RF}}(t) \cdot \cos \left[\omega_{\mathrm{RF}} t+\theta(t)\right]+V_{\mathrm{BB}}(t)$, where $V_{\mathrm{RF}}(t)$ and $\theta(t)$ are amplitude and phase information of the RF driving signal, respectively, $\omega_{R F}$ is the angular frequency of the RF carrier signal, and $V_{\mathrm{BB}}(t)$ is the amplitude of the $\mathrm{BB}$ driving signal. When the MZM is biased at null point, the generated optical signals can be expressed as

$$
\begin{aligned}
& E_{\text {out }}(t)=-E_{o} J_{0}\left[m_{\mathrm{RF}}(t)\right] \sin \left[m_{\mathrm{BB}}(t)\right] \cdot \cos \left[\omega_{o} t\right] \\
& +E_{o} J_{1}\left[m_{\mathrm{RF}}(t)\right] \cos \left[m_{\mathrm{BB}}(t)\right] \cdot \cos \left[\left(\omega_{o} \pm \omega_{\mathrm{RF}}\right) t \pm \theta(t)\right]
\end{aligned}
$$

where $m_{\mathrm{RF}}(t) \triangleq \pi V_{\mathrm{RF}}(t) / 2 V_{\pi}$ and $m_{\mathrm{BB}}(t) \triangleq \pi V_{\mathrm{BB}}(t) / 2 V_{\pi}$ are modulation index (MI) of RF and BB signals, respectively. 
The $J_{n}$ is the Bessel function of the first kind with order $n$. Notably, optical sidebands with the order of higher than $J_{1}$ can be ignored due to weak modulation condition. After square-law detection using a photodiode (PD), the desired $\mathrm{BB}$ and RF signals are obtained and can be written as

$$
\begin{aligned}
i_{\mathrm{BB}}(t)= & \frac{1}{2} R E_{o}{ }^{2} J_{0}{ }^{2}\left[m_{\mathrm{RF}}(t)\right] \sin ^{2}\left[m_{\mathrm{BB}}(t)\right] \\
& +R E_{o}{ }^{2} J_{1}{ }^{2}\left[m_{\mathrm{RF}}(t)\right] \cos ^{2}\left[m_{\mathrm{BB}}(t)\right] \\
i_{\mathrm{RF}}(t)= & \frac{1}{2} R E_{o}{ }^{2} J_{1}{ }^{2}\left[m_{\mathrm{RF}}(t)\right] \cos ^{2}\left[m_{\mathrm{BB}}(t)\right] \\
& \cdot \cos \left[2 \omega_{\mathrm{RF}} t+2 \theta(t)\right]
\end{aligned}
$$

where $R$ is the PD responsivity. The frequency of the generated $\mathrm{RF}$ vector signal $\left(2 \omega_{\mathrm{RF}}\right)$ is two times that of the driving signal $\left(\omega_{\mathrm{RF}}\right)$. Note that the amplitude and phase information of the generated RF vector signal are $J_{1}^{2}\left[m_{\mathrm{RF}}(t)\right]$ and $2 \theta(t)$, respectively. Therefore, the amplitude $\left(V_{\mathrm{RF}}(t)\right)$ and phase information $(\theta(t))$ of the driving signal needs to be predistorted to achieve the desired RF vector signals after square-law photodetection. By properly predistortion of driving signals, the proposed scheme can support various kinds of modulation formats, including amplitude-shift keying (ASK), phase-shift keying (PSK), and quadrature-amplitude-modulation (QAM) signals. No optical filter is needed to separate BB and RF signals if $M$-ary PSK signals are utilized for RF signals [7], which is compatible with the current optical passive optical network (PON). Therefore, the BB and RF signals can be rewritten as

$$
\begin{aligned}
i_{\mathrm{BB}}(t)= & R E_{o}^{2}\left[\frac{J_{0}^{2}\left(m_{\mathrm{RF}}\right)}{2}-J_{1}^{2}\left(m_{\mathrm{RF}}\right)\right] \sin ^{2}\left[m_{\mathrm{BB}}(t)\right] \\
& +R E_{o}^{2}{J_{1}}^{2}\left(m_{\mathrm{RF}}\right) \\
i_{\mathrm{RF}}(t)= & \frac{1}{2 R E_{o}^{2} J_{1}^{2}\left(m_{\mathrm{RF}}\right)} \cdot\left\{1-\sin ^{2}\left[m_{\mathrm{BB}}(t)\right]\right\} \\
& \cdot \cos \left[2 \omega_{\mathrm{RF}} t+2 \theta(t)\right]
\end{aligned}
$$

where $m_{\mathrm{RF}}$, i.e., $m_{\mathrm{RF}}(t)=m_{\mathrm{RF}}$, is constant if $M$-ary PSK signals are utilized. Hence, for BB signals, the $R E_{o}{ }^{2} J_{1}^{2}\left(m_{\mathrm{RF}}\right)$ term can be easily removed by using an electrical DC block. From (4), the BB signal power and the interference power induced by RF signals are proportional to $1 / 4 J_{0}^{4}\left(m_{\mathrm{RF}}\right) \sin ^{4}\left[m_{\mathrm{BB}}(t)\right]$ and $J_{1}^{4}\left(m_{\mathrm{RF}}\right) \sin ^{4}\left[m_{\mathrm{BB}}(t)\right]$, respectively. Therefore, the $\mathrm{BB}$ signal-to-interference ratio $\left(\mathrm{SIR}_{\mathrm{BB}}\right)$ can be defined as

$$
\operatorname{SIR}_{\mathrm{BB}}=\frac{J_{0}^{4}\left(m_{\mathrm{RF}}\right)}{4 J_{1}^{4}\left(m_{\mathrm{RF}}\right)} \text {. }
$$

For the RF signal, the desired RF signal power and the interference power induced by the BB signal are proportional to $1 / 4 \cdot J_{1}^{4}\left(m_{\mathrm{RF}}\right)$ and $1 / 4 J_{1}^{4}\left(m_{\mathrm{RF}}\right) \sin ^{4}\left[m_{\mathrm{BB}}(t)\right]$, respectively. We can define the RF signal-to-interference ratio $\left(\mathrm{SIR}_{\mathrm{RF}}\right)$ as

$$
\operatorname{SIR}_{\mathrm{RF}}=\sin ^{-4}\left[m_{\mathrm{BB}}(t)\right] \text {. }
$$

Fig. 2 shows $\operatorname{SIR}_{\mathrm{BB}}$ versus $m_{\mathrm{RF}}$ and $\mathrm{SIR}_{\mathrm{RF}}$ versus $m_{\mathrm{BB}}(t)$. Since the interference is in-band, it cannot be removed by electrical filters. As $m_{\mathrm{RF}}$ and $m_{\mathrm{BB}}(t)$ are less than 0.25 , both SIR $\mathrm{RF}$ and SIR $_{\mathrm{BB}}$ are more than $20 \mathrm{~dB}$. In the following experiment, both $m_{\mathrm{RF}}$ and $m_{\mathrm{BB}}(t)$ are confined to 0.07 to keep both SIR $\mathrm{BB}$ and SIR $_{\mathrm{RF}}$ above $40 \mathrm{~dB}$. Therefore, the system impairments are dominated by the uneven frequency response and high noise of

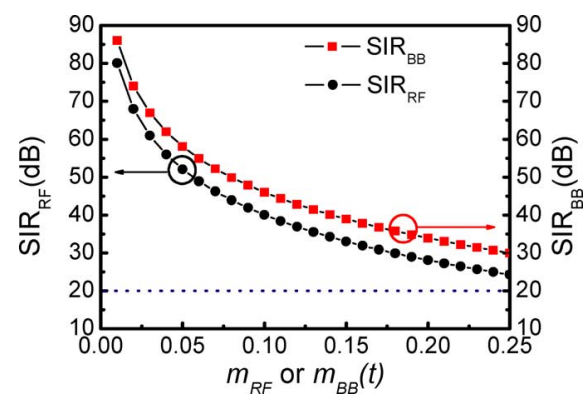

Fig. 2. $S_{R F}$ and $S I R_{B B}$ versus modulation index of $B B$ and $R F$, respectively.

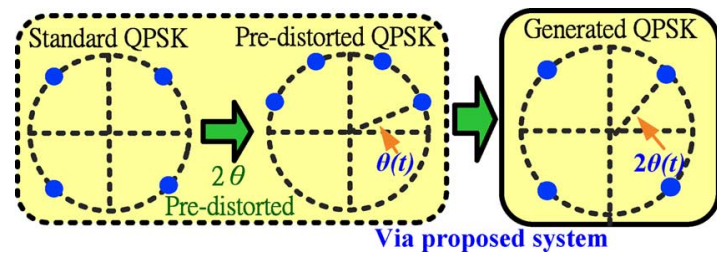

Fig. 3. Principle of predistorted scheme of the QPSK format.

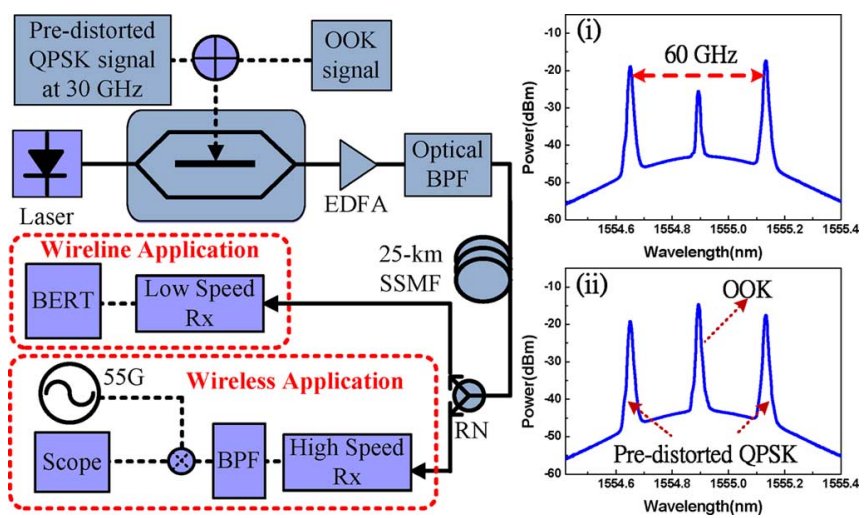

Fig. 4. Experimental setup of the proposed system. The optical spectrums (i) without OOK signal, (ii) with OOK signal (EDFA: erbium-doped fiber amplifier; BPF: bandpass filter; RN: remote node; BERT: bit-error-rate tester).

60-GHz components, instead of interference between BB and RF signals at large $m_{\mathrm{RF}}$ and $m_{\mathrm{BB}}(t)$.

In this letter, the $60-\mathrm{GHz}$ QPSK signal is experimentally demonstrated. Fig. 3 shows the principle of predistorted scheme of the QPSK format. The phases information of the predistorted QPSK signals $(\theta(t))$ which constellations are situated in the first and second quadrant are half of those of the generated QPSK signals $(2 \theta(t))$. After square-law photodetection via the DSB-CS modulation, the standard QPSK signal with frequency doubling is achieved.

\section{EXPERIMENTAL SETUP AND RESULTS}

Fig. 4 shows the experimental setup to verify the feasibility of the proposed system. A $2.5-\mathrm{Gb} / \mathrm{s}$ predistorted QPSK signal with a $2.5-\mathrm{GHz}$ center frequency is generated by an arbitrary waveform generator [(AWG) Tektronix AWG7102] with a Matlab program. After the AWG, the predistorted QPSK signal is up-converted to $30 \mathrm{GHz}$ using an electrical mixer. The $30-\mathrm{GHz}$ predistorted QPSK signal is combined with a BB 1.25-Gb/s OOK signal and sent into a single-electrode 

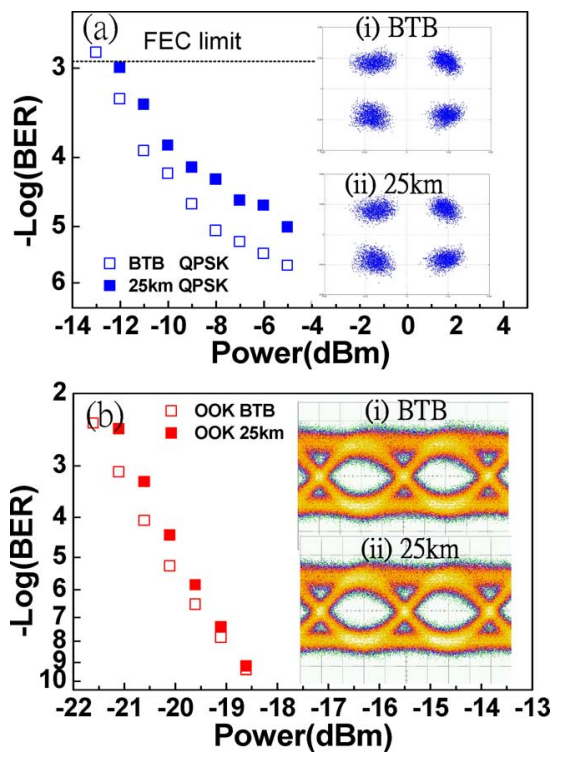

Fig. 5. BER curves of (a) QPSK signal, (b) OOK signal (FEC: forward-error correction).

MZM. Before the transmission of 25-km SSMF, the generated optical signal is amplified using an erbium-doped fiber amplifier and then passes through an optical bandpass filter to remove the amplified spontaneous emission (ASE) noise. At the receiver side, an optical coupler is employed to separate the optical power of the hybrid signal for different applications. For wireless applications, a standard QPSK signal with a center frequency at $60 \mathrm{GHz}$ is generated after square-law PD detection. To realize QPSK demodulation, the received QPSK signal is down-converted to $5 \mathrm{GHz}$ and captured by digital oscilloscope (Tektronix DPO 71254). An offline program using a Matlab program is used to demodulate and analyze the QPSK signal. The demodulated process includes synchronization and the bit-error-rate (BER) estimation. For wireline applications, the BB OOK signal is directly detected using a commercial $1.25-\mathrm{Gb} / \mathrm{s}$ photoreceiver and sent into a BER tester. A $60-\mathrm{GHz}$ DSB-CS optical spectrum with carrier suppression is observed while the BB OOK signal is turned OFF, as shown in inset (i) of Fig. 4. Only $30-\mathrm{GHz}$ transmitter bandwidth is required in this experimental setup. While the BB OOK signal is turned ON and combined with the predistorted QPSK signal, a double-sideband optical spectrum with OOK-modulated carrier is obtained as shown in inset (ii) of Fig. 4.

Due to the power limitation of available electrical RF amplifier in our laboratory, only $m_{\mathrm{RF}}=0.07$ can be achieved in this experiment. Therefore, both $m_{\mathrm{RF}}$ and $m_{\mathrm{BB}}(t)$ of 0.07 are used to verify the concept of the proposed system. According to the theoretical analysis, the mutual interference effects can be ignored. Fig. 5(a) shows the BER curves and constellations of QPSK signal. For the RF QPSK signal, the BER performance is calculated from the measured modulation error ratio [1]. The constellation diagrams of the recovered QPSK signal at BTB and after transmission are shown in the inset (i) and (ii) of Fig. 5(a). After transmission of 25-km SSMF, 4-dB receiver power penalty is observed at a BER of $10^{-5}$. The RF receiver power penalty comes from timing walk-off between the two optical sidebands with different optical frequencies [8].

Fig. 5(b) shows the BER curves and eye diagrams of the BB OOK signal. For the BB OOK signal, the receiver sensitivity at a BER of $10^{-9}$ is $-19 \mathrm{dBm}$ for BTB case. After 25-km SSMF transmission, the power penalty at a BER of $10^{-9}$ can be ignored.

\section{CONCLUSION}

Simultaneous generation and transmission of $60-\mathrm{GHz}$ $2.5-\mathrm{Gb} / \mathrm{s}$ QPSK and BB 1.25-Gb/s OOK signals using a single-electrode MZM was experimentally demonstrated in this study. Frequency doubling and multilevel modulation format was achieved using DSB-CS modulation scheme with a novel signal predistortion method. No narrowband optical filter was required at remote node to separate the $\mathrm{BB}$ OOK signal and $\mathrm{RF}$ QPSK signal for different applications, which is compatible with the current PON system. After transmission of $25-\mathrm{km}$ SSMF, 4-dB receiver power penalty which came from the timing walk-off between two optical sidebands was observed in the 60-GHz QPSK signal while a negligible receiver power penalty was observed in the BB OOK signal.

\section{REFERENCES}

[1] C. K. Weng, Y. M. Lin, and W. Way, "Radio-over-fiber 16-QAM, $100-\mathrm{km}$ transimission at $5 \mathrm{~Gb} / \mathrm{s}$ using DSB-SC transmitter and remote heterodyne detection," J. Lightw. Technol., vol. 26, no. 6, pp. 643-653, Mar. 15, 2008.

[2] V. Urick, J. Qiu, and F. Bucholtz, "Wide-band QAM-over-fiber using phase modulation and interferometric demodulation," IEEE Photon. Technol. Lett., vol. 16, no. 10, pp. 2374-2376, Oct. 2004.

[3] R. Sambaraju, V. Polo, J. L. Corral, and J. Marti, “Ten gigabits per second 16-level quadrature amplitude modulated millimeter-wave carrier generation using dual-drive Mach-Zehnder modulators incorporated photonic vector modulator," Opt. Lett., vol. 33, pp. 1833-1835, Aug. 2008.

[4] Y. Zhang, K. Xu, R. Zhu, J. Q. Li, X. B. Hong, and J. T. Lin, "Photonic generation of M-QAM/M-ASK signals at microwave/millimeter-wave band using dual-drive Mach-Zehnder modulatorswith unequal amplitudes," J. Lightw. Technol., vol. 26, no. 15, pp. 2604-2610, Aug. 1, 2008.

[5] Z. Jia, J. Yu, A. Chowdhury, G. Ellinas, and G. K. Chang, "Simultaneous generation of independent wired and wireless services using a single modulator in millimeter-wave-band radio-over-fiber systems," IEEE Photon. Technol. Lett., vol. 19, no. 20, pp. 1691-1693, Oct. 15, 2007.

[6] J. Kim, Y. Kang, Y. Chung, and K. Choi, "Development and RF characteristics of analog $60-\mathrm{GHz}$ electroabsorption modulator module for RF/optic conversion," IEEE Trans. Microw. Theory Tech., vol. 54, no. 2, pp. 780-787, Feb. 2006.

[7] P. T. Shih, C. T. Lin, W. J. Jiang, Y. H. Chen, Y. H. Chen, J. Chen, and S. Chi, "Hybrid access network integrated with wireless multilevel vector and wired baseband signals using frequency doubling and no optical filtering," IEEE Photon. Technol. Lett., vol. 21, no. 13, pp. 857-857, Jul. 1, 2009.

[8] J. Ma, J. Yu, C. Yu, X. Xin, J. Zeng, and L. Chen, "Fiber dispersion influence on transmission of the optical millimeter-waves generated using LN-MZM intensity modulation," J. Lightw. Technol., vol. 25, no. 11, pp. 3244-3256, Nov. 2007. 